\title{
Submitted: 11.2019 Multimodality imaging in a diagnostic and therapeutic Accepted: process of a patient with infective endocarditis
} 02.02.2020

Published: 31.03.2020

\section{Keywords}

infective endocarditis, multimodality

imaging, osteodiscitis, endocarditis team, echocardiography

\author{
Maria Binkiewicz-Orluk¹, Marcin Konopka', \\ Katarzyna Sierakowska-Sitkiewicz², Wojciech Król' ${ }^{1}$, \\ Leszek Królicki ${ }^{3}$, Marek Kuch², Wojciech Braksator ${ }^{1}$ \\ ${ }^{1}$ Department of Sports Cardiology and Noninvasive Cardiovascular Imaging, Medical \\ University of Warsaw, Warsaw, Poland \\ ${ }^{2}$ Department of Cardiology, Hypertension and Internal Diseases, Medical University of Warsaw, \\ Poland, Mazovia Brodno Hospital, Warsaw, Poland \\ ${ }^{3}$ Department of Nuclear Medicine, Medical University of Warsaw, Warsaw, Poland \\ Correspondence: Maria Binkiewicz-Orluk, Mazowiecki Szpital Bródnowski, Kondratowicza 8, \\ 03-242 Warsaw, Poland, tel.: +48 663 357 333,e-mail: maria.binkiewicz@gmail.com
}

DOI: $10.15557 / \mathrm{JoU} .2020 .0010$

\begin{abstract}
Despite the fact that more and more guidelines that systematize our knowledge are being published, infective endocarditis remains a considerable diagnostic and therapeutic challenge. This is partially caused by the variable course of the disease process and numerous potential complications with high mortality. We present a case of a 48 -year-old police officer with an inflammatory process within the aortic valve that led to the development of peripheral emboli in the central nervous system, kidney, spleen and within the lumbosacral spine. The complexity of the clinical situation required the cooperation of various medical specialists and combined use of various imaging methods, including ultrasound. This ultimately enabled the correct diagnosis to be established and had a significant impact on the planning and monitoring of the diagnostic process.
\end{abstract}

\section{Case description}

The patient was a 48-year-old professionally active police officer. He was admitted to hospital due to an episode of syncope that occurred while driving a car. At admission, the patient's condition was good, with no irregularities on physical examination. He reported recurrent subfebrile states and body weight loss. Laboratory tests demonstrated significantly elevated inflammation markers (CRP $=75 \mathrm{mg} / \mathrm{L}$ ) and considerably increased D-dimer values $(21,000 \mathrm{ng} / \mathrm{L})$. Computed tomography (CT) of the head revealed a large hypodense region in the right brain hemisphere, corresponding to ischemic stroke (Fig. 1).

CT angiography of the thoracic arteries ruled out pulmonary embolism, but abdominal CT showed hypodensities within the spleen and left kidney (Fig. 2).
Because the patient developed fever $\left(40^{\circ} \mathrm{C}\right)$, the inflammatory parameters were elevated and there were peripheral infarction foci, transthoracic echocardiography was ordered. This examination turned out to be crucial for establishing a diagnosis and directed further therapeutic steps. Echocardiography revealed normal size and geometry of the cardiac cavities, and ejection fraction was estimated at $70 \%$. Of observed abnormalities, attention was drawn to a mobile, 23-millimeter structure linked with aortic valve cusps (right and non-coronary), indicative of bacterial vegetation (Fig. $3 \mathrm{~A}, \mathrm{C}$ and D). As a consequence of an ongoing inflammatory process, considerable aortic regurgitation developed, which was illustrated as holodiastolic flow reversal in the descending aorta with high enddiastolic velocities (>0.2 m/s) (Fig. 3 B). Moreover, there was also a slight amount of fluid in the pericardial sac (up to $7 \mathrm{~mm}$ ). 


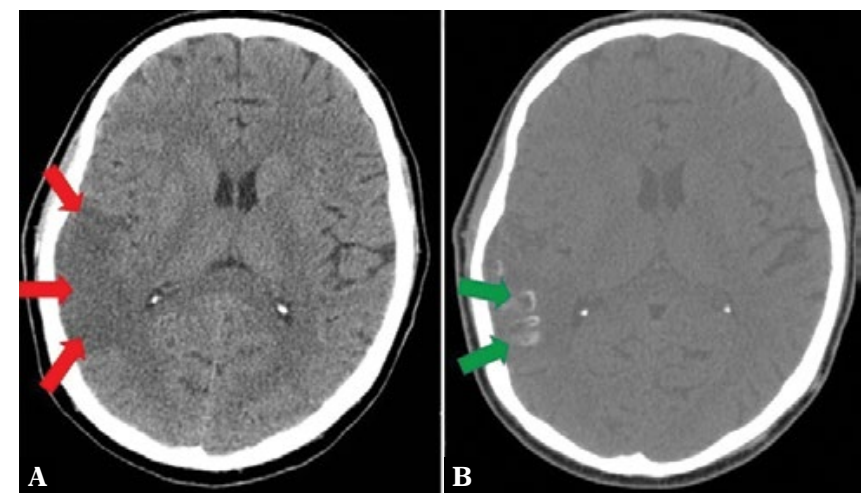

Fig. 1. Computed tomography of the head without contrast-enhancement. A. Red arrows indicate a large, hypodense region corresponding to an ischemic focus within the temporal lobe. B. Follow-up after 25 days of treatment: a cortical-subcortical focus of ischemic stroke within the right temporal lobe with calcifications on the cortex outlines
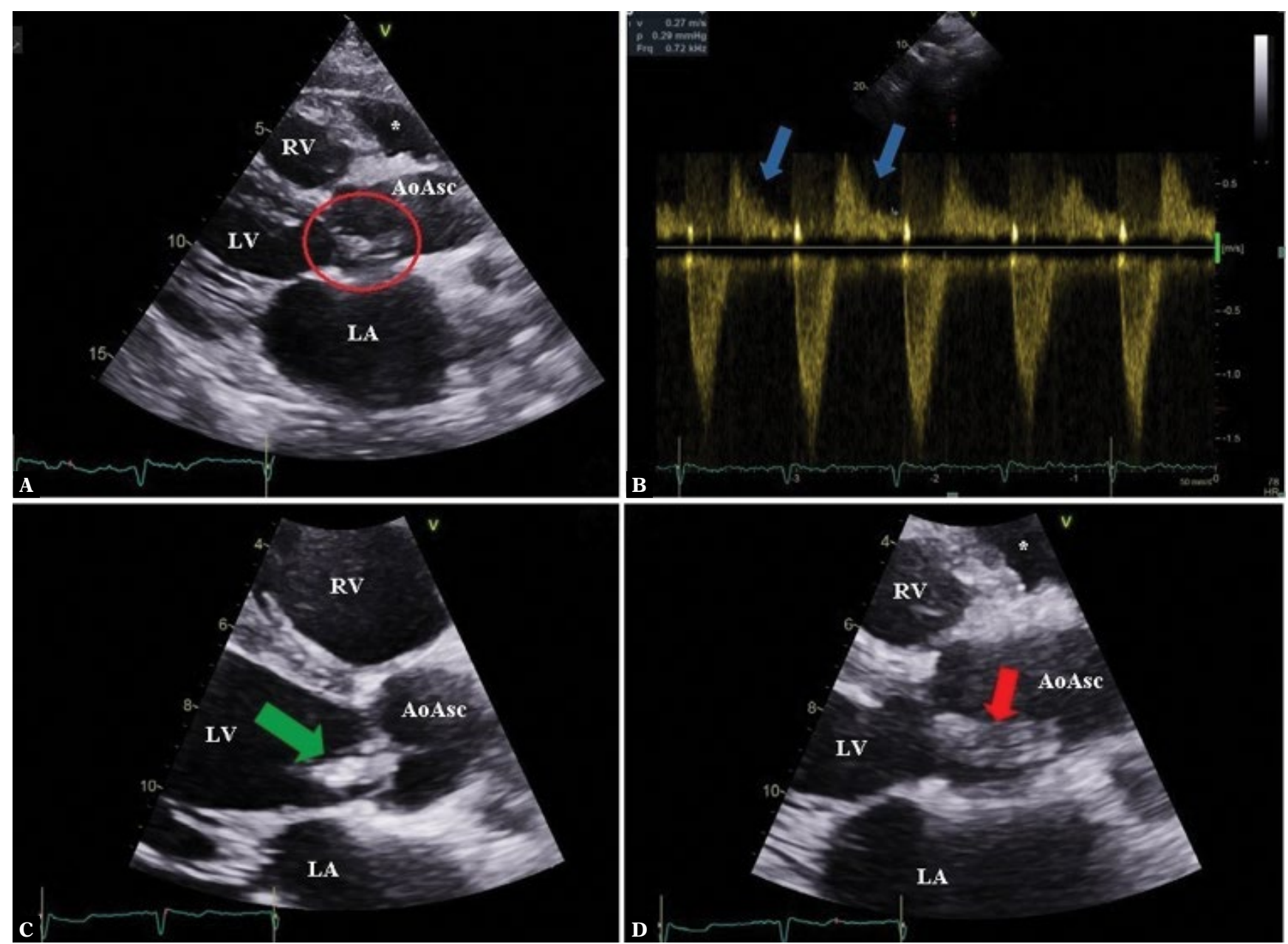

Fig. 3. Transthoracic echocardiography. A. Sternal, long-axis view: a structure linked with aortic valve cusps, corresponding to a vegetation. B. Suprasternal view with blood flow assessment in the descending aorta using pulsed Doppler: arrows indicate holodiastolic flow indicating considerable regurgitation. $\mathbf{C}$ and $\mathbf{D}$. Parasternal, long-axis views, zoomed aortic valve (ZOOM): changes in the position of the vegetation depending on the cardiac cycle: systole (C), diastole (D). AoAsc-ascending aorta, LA - left atrium, LV-left ventricle, $R V$-right ventricle, * - pericarcial fluid
According to the modified Duke infective endocarditis criteria, the diagnosis of this condition was certain at this stage of the diagnostic process: the patient met one major criteria (evidence of vegetation on echocardiography) and two minor criteria (fever $>38^{\circ} \mathrm{C}$ and peripheral embolism in the central nervous system [CNS], kidney and spleen $)^{(1)}$. 


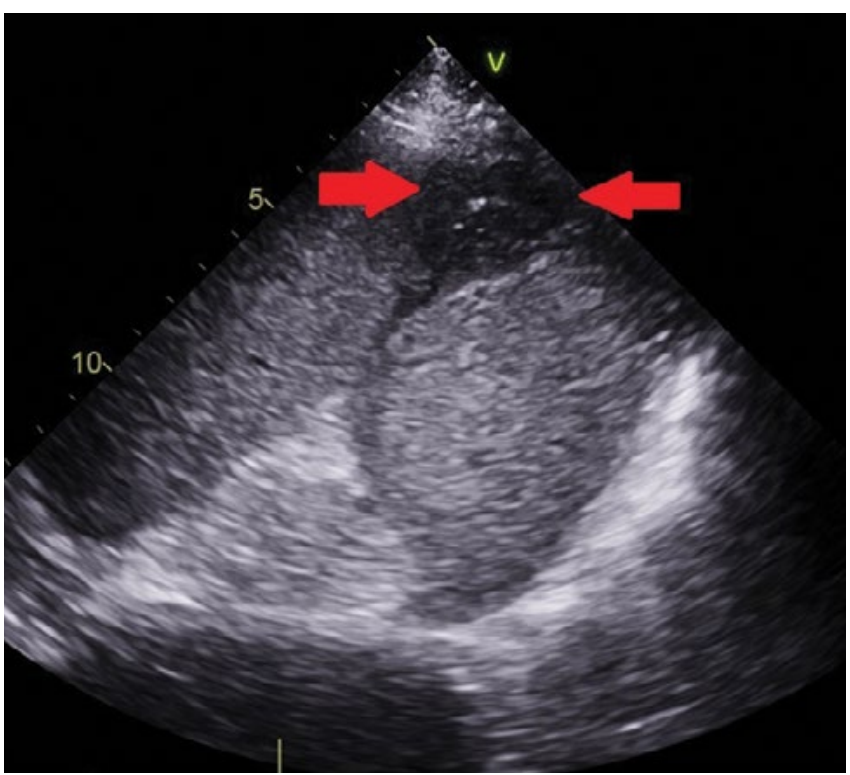

Fig. 4. Abdominal ultrasound with a sector echocardiography transducer: a triangular area of infarction in the spleen (also seen in computed tomography of the abdomen shown in Fig. 2A)

Moreover, a scan with an echocardiography sector probe showed a hypoechoic, triangular region within the spleen, corresponding with the infarction focus described previously in the abdominal CT report (Fig. 4).
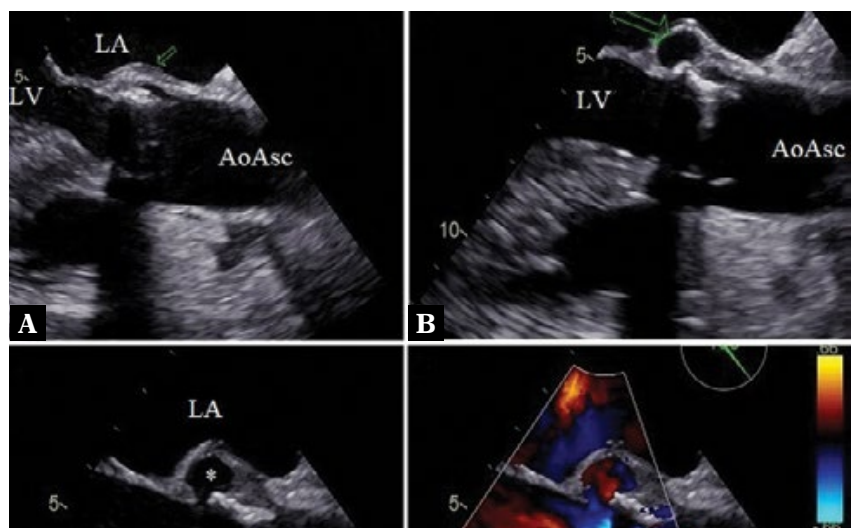

LV
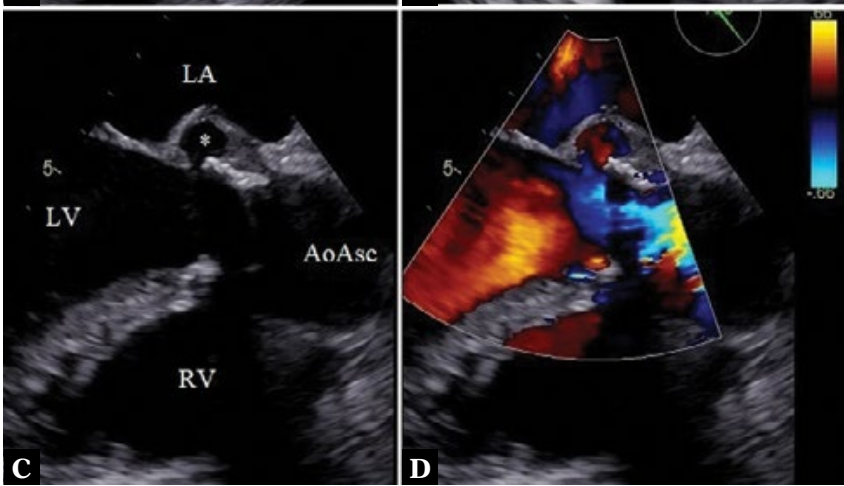

Fig. 5. Echocardiography, high transesophageal view, long axis. A. 35 days after aortic valve replacement: a thickening seen at the site of valvular prosthesis implantation. B. 54 days after surgery: a slight paravalvular aneurysm is seen at the site of previous wall thickening. C. Clearly visible inflow gate: the aneurysm communicates with the left ventricular outflow tract. D. Color Doppler presents evidence of flow within the lesion. Abbreviations: AoAsc - ascending aorta, $L A$ - left atrium, $L V$ - left ventricle, $R V$ - right ventricle, * - a space corresponding to the paravalvular aneurysm
Owing to the size of the vegetation and the presence of embolic complications (including CNS involvement), the patient was deemed eligible for an urgent surgery for aortic valve replacement. The surgery proceeded without complications. At the same time, the results of preoperative blood cultures arrived. They revealed infection with Streptococcus gallolyticus which, according to some authors, accounts for even $6 \%$ of all etiological factors in infective endocarditis $(\mathrm{IE})^{(3,4)}$. Cultures of the native valve showed Streptococcus mutans, which belongs to the same group of bacteria.

Taking into account the fulminant course of the disease as well as the opinion of the operators and clinical pharmacologist, the following combined antibiotic therapy was ordered in this specific case: crystalline penicillin (6 weeks), vancomycin (6 weeks) and gentamycin (2 weeks). This is not a typical regimen as per guidelines, which recommend therapy with two or even one antibiotic in similar cases ${ }^{(1,2)}$. Moreover, the patient had a carious tooth removed as it might have initiated the inflammatory process.

Despite this pharmacological management, which initially resulted in CRP decline to $21 \mathrm{mg} / \mathrm{L}$ (on day 23. after surgery), this parameter increased again to $62 \mathrm{mg} / \mathrm{L}$ (on days 33-35 after surgery). No pathogens were found on subsequent blood cultures. The diagnostic process was resumed to rule out possible foci of infection.

Transesophageal echocardiography (TEE) showed a normally functioning bioprosthesis with no clear evidence of vegetation or paravalvular leaks. Thickened aortic wall drew attention (Fig. $5 \mathrm{~A}$ ). This area was verified in a subsequent transesophageal examination where a slight paravalvular aneurysm was found (Fig. 5 B).

Moreover, because of persisting back pain, magnetic resonance imaging was ordered and demonstrated inflammatory changes suggestive of osteodiscitis at the L5/S1 level (Fig. 6). Due to the lack of clear guidelines regarding the manner and duration of treatment in similar $\operatorname{cases}^{(9,10)}$, a clinical pharmacologist was asked for advice and a center specializing in the treatment of bone infections was consulted. Following their instructions, empirical antibiotic therapy was implemented; it consisted of: meropenem (6 weeks), crystalline penicillin (6 weeks) and gentamycin ( 2 weeks). After the conclusion of intravenous treatment, it was decided to continue outpatient antibiotic therapy until CRP normalization ${ }^{(9)}$.

In order to search for other infection foci and to absolutely exclude the presence of inflammation within the newly implanted aortic valve prosthesis, positron emission tomography-computed tomography with radiolabelled leukocytes (PET-CT) was performed. Increased tracer accumulation was observed within the palatine tonsils, at the L5/S1 level of the spine and within the post-infarction area in the spleen (Fig. 7). The tracer did not accumulate within the implanted valvular prosthesis.

Following PET-CT, it was decided to perform urgent tonsillectomy. 

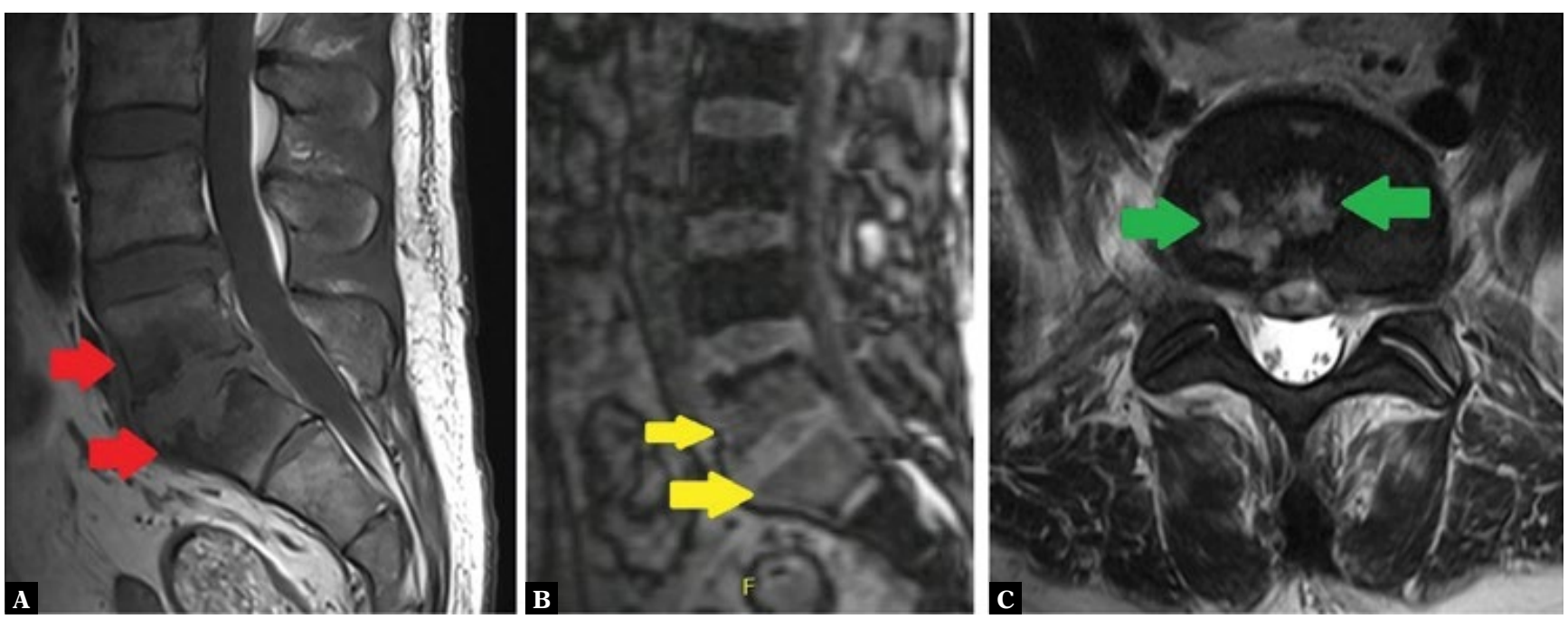

Fig. 6. Magnetic resonance imaging of the lumbosacral spine. A. A low-signal region on T1-weighted images within the L5 and S1 vertebral bodies. $\mathbf{B}$ and $\mathbf{C}$. Arrows indicate areas of increased signal on T2-weighter images

The patient was discharged in an overall good condition. As agreed by the therapeutic team consisting of a cardiologist, a specialist in infectious diseases and a clinical pharmacologist, the patient continued antibiotic therapy on an outpatient basis for 4 months. He remains in contact with a cardiology clinic. Outpatient followup laboratory tests show persisting, unelevated values of inflammation markers (CRP), and a follow-up abdominal ultrasound demonstrated regression of the infarction foci previously seen within the kidney and spleen. The patient returned to full performance and resumed professional activity.

\section{Discussion}

Because of its variable course, infective endocarditis remains an insidious disease, the diagnosis and treatment of which are a challenge for physicians of various specialties. The case presented above required cooperation of cardiologists, cardiac surgeons, neurologists, specialists in infectious diseases, pharmacologists and radiologists. It therefore seems legitimate to form "Endocarditis Teams," as mentioned in the ESC guidelines ${ }^{(1)}$.

According to some sources, mortality among patients with IE reaches even $26 \%{ }^{(3,6)}$ and is particularly associated with the risk of complications in the form of septic peripheral embolism (including CNS strokes) and acute heart failure due to valvular insufficiency. Early suspicion and confirmation of the diagnosis enable prompt therapeutic decisions (antibiotic therapy, surgical intervention), which significantly improves prognosis.

Apart from the vigilant observation by clinicians, modern imaging methods used in combination and supplementing one another (so-called multimodality imaging) are also invaluable in such cases as they offer the complete clinical picture ${ }^{(7)}$. The usefulness of multimodality imaging has been acknowledged in the modified Duke criteria from 2015. They were compared with the previous criteria from 1992, and the so-called major criteria (i.e. positive blood culture, echocardiographic evidence defined as the presence of vegetation, abscess, pseudoaneurysm, intracardiac fistula; valvular perforation or aneurysm; new partial dehiscence of prosthetic valve) were supplemented with further two:

- evidence of an infective process on PET/CT or single-photon emission computed tomography (SPECT);

- definite lesions on cardiac CT.

The minor criteria remained practically unchanged apart from the event when echocardiography suggests IE but the major criteria are not met; it is then necessary to broaden the diagnostic process to include new imaging methods ${ }^{(1)}$

Transthoracic echocardiography still remains the gold standard in IE diagnosis. It should be performed as the first-choice examination in each patient with a suspicion of this disease ${ }^{(1)}$. Apart from confirming the presence of vegetation, the examination also provides information about anatomic and functional conditions of the heart, makes it possible to estimate ejection fraction and enables assessment of valvular function.

Transesophageal echocardiography, which supplements and broadens the transthoracic examination, should be the next diagnostic step. It holds a rather special position in preoperative diagnosis owing to its technical capabilities enabling precise visualization of altered valvular morphology and estimation of the size of vegetation (which is one of the factors deciding about the urgency of intervention). Recently, the intraoperative role of TEE has been emphasized more and more frequently to evaluate for paravalvular tissue complications at the site of the prosthesis; it is an important prognostic factor that affects overall mortality ${ }^{(7)}$. 

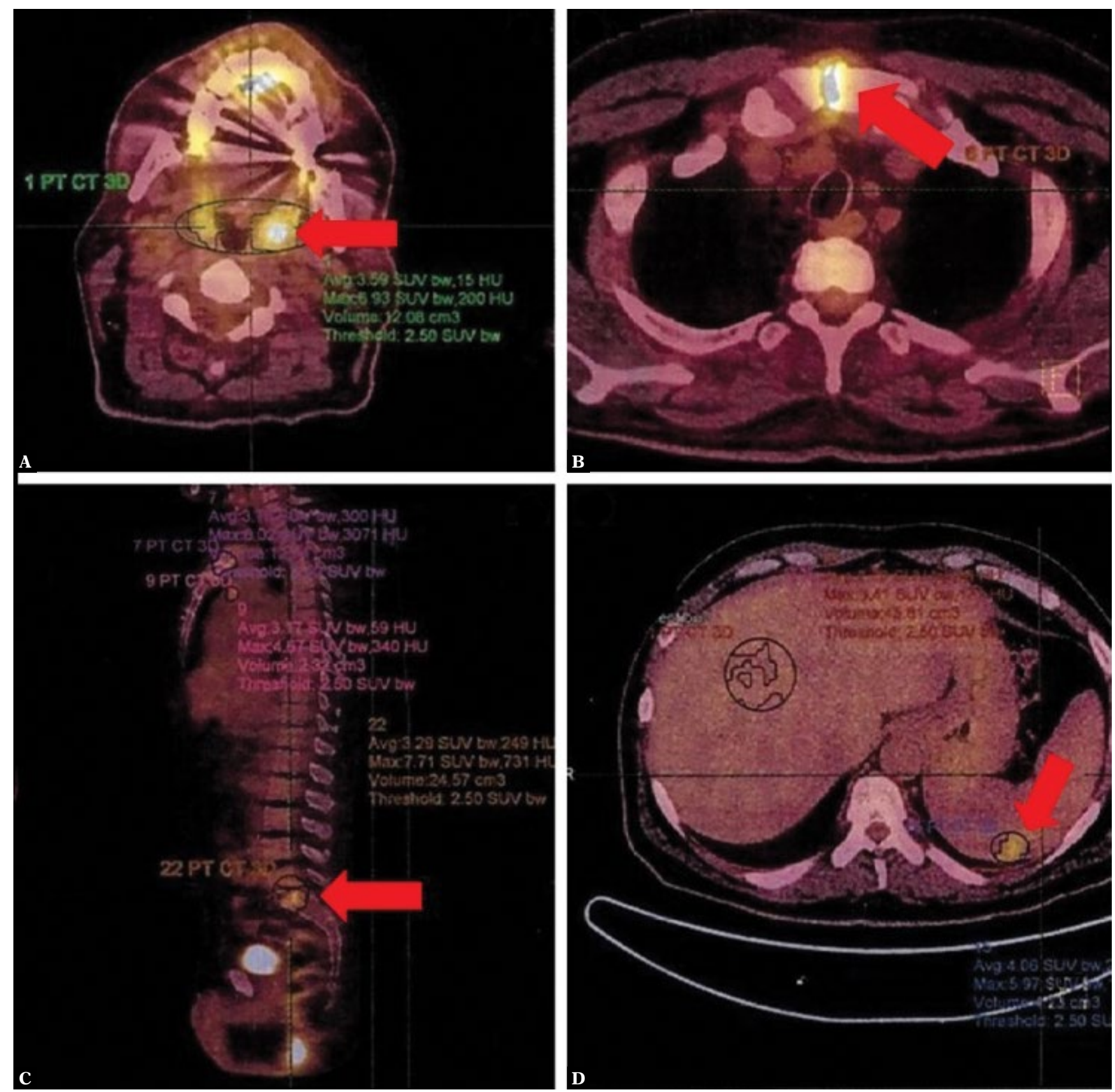

Fig. 7. 18-fluorodeoxyglucose positron emission tomography. A. Enhanced tracer accumulation within the palatine tonsils. B. Enhanced and diffuse tracer accumulation in the superior part of the mesosternum: the findings correspond to the healing process after sternotomy. C. Diffuse area of enhanced tracer accumulation within the L5/S1 vertebral bodies: the entire image suggests inflammatory nature of the lesions. D. A peripheral, subcapsular region of enhanced tracer accumulation in the spleen. It corresponds to an infarction focus seen also on abdominal ultrasound (Fig. 4) and computed tomography (Fig. 2A)

Computed tomography is useful in postoperative monitoring as it enables evaluation for local complications forming around the prosthesis (abscess, paravalvular aneurysm) and assessment of sternum and soft tissue healing. In the discussed patient, ultrasonographic assessment of soft tissues, which is more available and less burdensome, was a valuable supplementation of computed tomography during the postoperative healing process. It was conducted to assess suspicious fluid collections around the sternum, which were ultimately found to be a normal healing process of the postoperative wound. Furthermore, conventional abdominal ultrasound enabled monitoring of infarction changes in the kidney and spleen; evidence of their gradual regression aided decision-making concerning splenectomy, which was ultimately abandoned.

A poorly available yet very helpful in IE therapy monitoring is positron emission tomography (PET-CT). It enables one to locate all ongoing inflammatory processes owing 
to the accumulation of a tracer substance in these areas. Performing this examination in the patient with growing CRP values despite broad-spectrum antibiotic therapy helped to definitely exclude inflammation at the valvular prosthesis and unambiguously confirm the inflammatory etiology of the observed spinal changes.

\section{Conclusion}

1. IE is a disease with a complex clinical picture. Its assessment requires multimodality imaging.

2. Thanks to multimodality imaging, therapeutic decisions can be made promptly and accurately, and the treatment process can be monitored, which entails better prognosis.

3. It is necessary to search for new imaging methods and formulate appropriate diagnostic protocols.

\section{Conflict of interest}

Authors do not report any financial or personal connections with other persons or organizations, which might negatively affect the contents of this publication and/or claim authorship rights to this publication.

5. Murillo O, Grau I, Gomez-Junyent J, Cabrera C, Ribera A, Tubau F et al.: Endocarditis associated with vertebral osteomyelitis and septic arthritis of the axial skeleton. Infection 2018; 46: 245-251.

6. Hase R, Otsuka Y, Yoshida K, Hosokawa N: Profile of infective endocarditis at a tertiary-care hospital in Japan over a 14-year period: characteristics, outcome and predictors for in-hospital mortality. Int J Infect Dis 2015; 33: 62-66.

7. Gálvez-Acebal J, Almendro-Delia M, Ruiz J, de Alarcón A, MartínezMarcos FJ, Reguera JM et al.: Influence of early surgical treatment on the prognosis of left-sided infective endocarditis: a multicenter cohort study. Mayo Clin Proc 2014; 89: 1397-1405.

8. Martí-Bonmatí L, Sopena R, Bartumeus P, Sopena P: Multimodality imaging techniques. Contrast Media Mol Imaging 2010; 5: 180-189.

9. Gouliouris T, Aliyu SH, Brown NM: Spondylodiscitis: update on diagnosis and management. J Antimicrob Chemother 2010; 65: iii11-24.

10. Maffulli N, Papalia R, Zampogna B, Torre G, Albo E, Denaro V: The management of osteomyelitis in the adult. Surgeon 2016; 14: 345-360. 